\title{
RATE ALLOCATION FOR SVC TRANSMISSION IN ENERGY-CONSTRAINED WIRELESS SENSOR NETWORKS
}

\author{
Seyedeh Zahra Sadri Tabaee Zavareh ${ }^{1}$ and Mahmood Fathy ${ }^{2}$ \\ ${ }^{1}$ Islamic Azad University Science and Research Branch, Tehran, Iran \\ z.sadriesrbiau.ac.ir \\ ${ }^{2}$ Computer Engineering Department, Iran University of Science and Technology, Tehran, \\ Iran \\ mahfathy@iust.ac.ir
}

\begin{abstract}
Due to the resource limitations, efficient multimedia transmission is still a challenging problem in Wireless Sensor Networks (WSNs). To achieve this goal, provisioning the required rate and quality of video stream along with limitations of sensor nodes should be considered. The Scalable Video Coding (SVC) is an efficient approach to support the graceful quality reduction and scalability of multimedia application thus we adopt it to encode video streams. In this paper, both Network Utility Maximization (NUM) and utility proportional optimization for scalable multimedia transmission in WSNs are addressed. Link congestion and energy scarcity of sensor nodes are considered as the constraints in optimization problems. To depict the characteristics of these applications accurately, staircase utility function is adopted. The non-concavity of utility function leads to the non-convex problem. To deal with the non-convexity of the problem, an approximation of the utility function is used. Finally, we propose two distributed algorithm to allocate the resources properly by solving NUM and utility proportional problems in energy-constraints WSNs. The simulation results in different scenarios show the efficiency and proper rate of convergence of our proposed algorithms.
\end{abstract}

\section{KEYWORDS}

Resource Allocation, Energy, Scalable Video Coding, Network Utility Maximization, Utility Proportional Fairness, Wireless Sensor Networks

\section{INTRODUCTION}

Wireless multimedia sensor networks are comprised of sensor nodes which equipped by low-cost hardware such as CMOS camera which can collate multimedia content such as video, audio and images from the environment. Emergence of these applications in WSNs has extended potential application range in WSNs from multimedia surveillance sensor networks to industrial process control. The energy deficiency and low bandwidth in sensor nodes along with quality of service requirements of multimedia applications in terms of bandwidth and energy consumption bring about new technical challenges in multimedia transmission in WSNs. In order to deliver these applications efficiently and guarantee the desired network lifetime for WSN, technical points in 
multimedia coding techniques, energy scarcity of sensor nodes and efficient bandwidth allocation should be considered.

The prominent point in multimedia transmission is to adjust rate and video quality of video streams in a way to deal with varying throughput of wireless channel. The SVC standard [1], [2] which is an extension to H.264/ AVC standard [3] leads to reach this goal. In fact, this coding technique provides scalable multimedia transmission and allows graceful quality reduction in the case of adverse condition in network. Based on enumerated advantages and also its wide applications in WSNs [4], we adopt it to encode video streams.

Sensor nodes require energy to perform their defined tasks such as sensing, transmitting and receiving data. The energy of wireless sensor nodes limits to their batteries and often substituting their batteries are infeasible. So, energy deficiency is one of the fundamental concerns in WSNs and power management play a prominent role in lifetime of a wireless sensor network [5]. In addition, multimedia applications like video streaming which are addressed in this paper, consume considerable amount of energy to be delivered efficiently. Moreover, these kinds of contents have high bandwidth demand and strict QoS requirements. Therefore, it seems worthy to investigate an approach to allocate resources including bandwidth and energy by optimization techniques properly.

Since the publication of the seminal paper [6] by Kelly et al. in 1998, a systematic method for providing efficient rate control called Optimization Flow Control (OFC) was proposed. Following this pioneering work, Low et al. in [7] established a general duality model to efficiently solve the rate allocation problem and proposed the Network Utility Maximization (NUM) framework. Based on this framework, bandwidth sharing problem has been exploited in communication networks with the aim of maximizing aggregate utility of sources. Hence, flow control based on optimization methods has been widely applied in wireless and wired networks [8]-[11]. Moreover, it has been extensively explored in wireless sensor networks [12]-[15].

The primary assumption in these works is that the utility function is increasing and strictly concave which makes this approach suitable for elastic traffic. Hence, this framework is not appropriate for multimedia applications, which their characteristics are often shown by nonconcave utility functions [8], [16]. This non-concavity causes non-convex NUM. Several works such as [8], [17] and [18] have addressed this issue. In [8], the authors eliminate the strict concavity condition and just consider continuously increasing utilities in problem definition. By introducing Utility Proportional Fairness (UPF), they proposed a new flow control algorithm with different rate adjustment approach that is more appropriate for real-time and multimedia applications. In [17], the problem of distributed rate allocation for inelastic flows is tackled. The conditions under which the standard price-based distributed algorithm can converge to the globally optimal rate by the assumption of non-concavity of utility function are investigated. Finally, an optimal distributed algorithm to allocate rates between elastic and inelastic flows is developed. In [18], staircase utility function for the utility of scalable multimedia streams is chosen. To deal with the non-convexity of the NUM problem, a smooth approximation of staircase utility function is used and a dual-based distributed algorithm for rate allocation is proposed.

The purpose of this paper is to allocate bandwidth among SVC streams with aim of maximizing total utility in WSNs. As energy plays a vital role in WSNs, we also consider a power dissipation model. Towards this, we formulate this problem in a NUM framework with bandwidth and energy constraints. Since the UPF is more appropriate for multimedia streams, we reformulate and solve the problem using it. We use a staircase function to show the characteristics of scalable multimedia streams. To tackle the non-concavity of such a function, we apply multimodal sigmoid approximation [18]. Finally, by solving NUM and UPF problems, we propose two dual- 
based distributed algorithms to allocate bandwidth and energy efficiently and fairly among streams of sensor nodes.

The rest of the paper is organized as follows. In Section 2, we describe the system model including utility and power model. We formulate resource allocation problem as an optimization problem in Section 3. In Section 4, we first solve the optimization problems. Then, we propose distributed algorithms which result from solving optimization problems. In order to verify the convergence of the algorithm, numerical results are depicted in Section 5. Finally, we conclude our paper in Section 6.

\section{SYSTEM MODEL}

We consider a static wireless video sensor network comprising of a set $S=\{1,2, \ldots, S\}$ of sensor nodes and a sink node. Sensor nodes collect visual data about phenomena of interest and report their sensing data to the sinks through a set $L=\{1,2, \ldots, L\}$ of wireless communication links. We assume each link $l$ has a constant capacity $c_{l}$ and interference issue is handled in PHY and MAC layer. Each sensor node $s$ is characterized by five parameters $\left\{L_{s}, x_{s}, m_{s}, M_{s}, U_{s}\left(x_{s}\right)\right\}$ where $x_{s}$ is the data rate of sensor node $s$ that should satisfy $m_{s} \leq x_{s} \leq M_{s}$, where $m_{s}$ and $M_{s}$ are the minimum and maximum transmission rate for $s$, respectively. The route $L_{s} \subseteq L$ is a subset of links, which connect sensor node $s$ to the sink. We suppose that there is a single path between each node and the sink. Also, we assume the route is defined by routing protocol in network layer. For each link $S_{l}=\left\{s \in S \mid l \subseteq L_{s}\right\}$ denotes the set of nodes that use link $l$. $U_{s}\left(x_{s}\right): R^{+} \rightarrow R$ is a utility function which denotes QoS performance of sensor node $s$. For the sake of simplicity, we define routing matrix $\boldsymbol{R} \in\{0,1\}^{L \times S}$ where the entry $R_{l s}=1$ if and only if $l \in L_{s}$ or $s \in S(l)$, and 0 otherwise.

\subsection{Utility Model}

As already mentioned, we address resource allocation for SVC encoded video streams in WSNs. In scalable video coding, a video stream is encoded in a layered scheme, a base layer and several enhancement layers. In this way, a sensor can adopt its rate and quality of video sequence according to the current network situation. As a result, when congestion occurs in a network, the video stream can be sent using low bit coded layers and the enhancement layers can be sent if their required bandwidth is accessible [1]. Hence, we can show the utility function for SVC encoded streams by staircase function (Figure 1). Here, the utility is increased only when a higher layer can be delivered due to increase in the available bandwidth. The staircase function is nonconcave and non-differentiable which makes the optimization problem non-convex, and its corresponding rate allocation optimization problem is usually hard to solve.

To deal with the non-convexity of the problem, an approximation of utility function called multimodal sigmoid approximation is proposed in [18]. This approximation is depicted in Figure 1. In order to construct the approximation of the staircase function, at the first, its domain is divided into non-overlapping intervals such that a step transition occurs in the middle of each interval. Then, by exploiting logistic function $F(x, \alpha, \beta)=\frac{1}{1+e^{-\alpha(x-\beta)}}$ each step transition is approximated. In logistic function, $\beta$ is the inflection point, i.e. for $x \prec \beta$, the function is convex and for $x \succ \beta$ it is concave. Furthermore, $\alpha \succ 0$ determines the sharpness of the curve and should be chosen large enough to appropriately capture the sharp transition of an increase in the 
International Journal of Computer Networks \& Communications (IJCNC) Vol.4, No.1, January 2012

utility level. The following equation is obtained at transition point $i$ where $U(x)=i$ jump to $U(x)=i+1[18]$ :

$$
\tilde{U}(x)=F(x, \alpha, k i)+i ; x \in\left[k i-\frac{k}{2}, k i+\frac{k}{2}\right]
$$

Where $\tilde{U}_{s}$ (.) denotes the approximation of the primary utility function and $\left[k i-\frac{k}{2}, k i+\frac{k}{2}\right]$ is the interval which transition $i$ occurs. Also, $k$ is the required rate increase to advance the utility $U$ by 1 . Here, we consider constant distance between each transition.

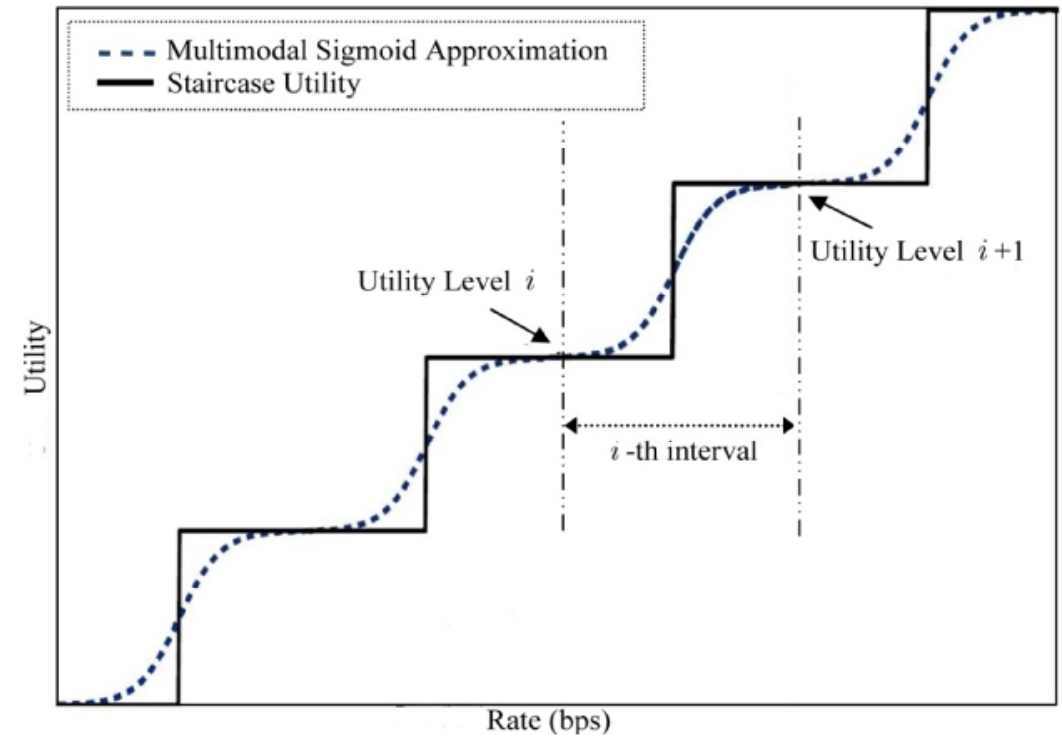

Figure 1.Staircase utility function (solid line) and its approximation (dashed line) for SVCencoded streams [18]

Combining all the intervals, the following approximated utility function was yielded

$$
\tilde{U}=\left\{\begin{array}{cc}
F(x, \alpha, k) & x \in\left[k-\frac{k}{2}, k+\frac{k}{2}\right] . \\
F(x, \alpha, k i)+i & x \in\left[k i-\frac{k}{2}, k i+\frac{k}{2}\right] . \\
\cdot & x \in\left[k N-\frac{k}{2}, k N+\frac{k}{2}\right] .
\end{array}\right.
$$

Where $\mathrm{N}$ denotes the number of intervals associated with $\mathrm{N}$ encoding layer.

The proposed approximation is discontinuous in overall, which can be solved by choosing $\alpha$ sufficiently large.

\subsection{Power Dissipation Model}

We use the power dissipation model proposed in [14] to consider energy consumption of sensor nodes. Since sensor nodes encounter much tighter energy constrains than sinks, we focus our power dissipation model on sensor nodes side and assume enough energy on sink side. Sensor 
nodes consume energy for performing their tasks such as sensing, transmitting and receiving data. They mainly consume their energy in communication process rather than computational process [19], so we ignore energy consumed in latter part. Hence, we consider $E^{s}$ for energy consumed in sensing, $E^{t}$ and $E^{r}$ as consuming energy in transmitting and receiving processes, respectively. Also, each sensor node $s$ has a limited initial energy $I_{s}$ and $\boldsymbol{I}$ is the vector of initial energies.

We suppose the identical power dissipation features for all sensor nodes and define a matrix $\boldsymbol{E} \in R_{+}^{\mathrm{S} \times \mathrm{S}}$ as follows

$$
E_{i s}= \begin{cases}E^{s}+E^{r} & \text { If node } i \text { is the origin of } \\
& \begin{array}{l}
\text { If node } i \text { is the } \\
E^{t}+E^{r}
\end{array} \\
& \begin{array}{l}
L_{s} \\
\text { Intermediate node of route }
\end{array} \\
0 & \text { If node } i \text { is not a part of route }\end{cases}
$$

The total consumed energy at node $s$ for a stream $x$ can be obtained from the following equation

$$
\mathcal{P}_{s}=E_{s} x
$$

Where $E_{s}$ is the $s$ th row of matrix $\boldsymbol{E}$.

Definition 1: The network lifetime $T$ is equal to minimum lifetime of sensor nodes, i.e. $T=\min _{s \in S} T_{s}[19]$.

We assumed $T_{d}$ as defined network lifetime for WSN which should be reached. In order to achieve $T_{d}$, each node should not consume energy more than $P_{s}^{\max }=\frac{I_{s}}{T_{d}}$. Let $\boldsymbol{p} \in R_{+}^{S}$ be the vector of maximum admissible energy consumption of each node. Therefore, to ensure $T_{d}$, the following energy constraint should be considered

$$
\boldsymbol{E} x \leq \boldsymbol{p}
$$

In the following section, we formulate the resource allocation problem in an optimization framework based on the presented utility and energy model.

\section{Problem Formulation}

The OFC approach is addressed the way of bandwidth sharing and rate allocation among multiple traffic flows over a shared network with regards to link capacity constraints. According to Kelly's work [6], source $s$ attains a utility $U_{s}\left(x_{s}\right)$ if it sends at rate $x_{s}$ and used to quantify its benefit in terms of QoS. The main objective in this rate allocation problem which is based on optimization problem is to maximize the aggregate utility functions $\sum_{s} U_{s}\left(x_{s}\right)$ with a link capacity constraint $\sum_{s} R_{l s} x_{s} \leq c_{l}$. The constraint says that the aggregate source rates at link $l$ should not exceed its capacity. A distributed resource allocation algorithm is obtained by solving the optimization problem. It should be noticed that the sink in WSNs is responsible to gather sensed data from sensor nodes and plays no role in network utility. 
As the main purpose in OFC is to maximize network utility, it sometimes treats to some applications unfairly. Actually, it prefers to allocate bandwidth to application which has low bandwidth demand and its utility function increases rapidly. In contrast, an application with a high bandwidth demand and slow upward trend in its utility function is treated poorly; because, such an allocation has a small impact on increasing total utility.

In order to overcome this issue, Utility Proportional Fairness (UPF) is proposed [8]. This approach revisits the flow control problem and gives special consideration to real-time applications which are sensitive to transmission bandwidth. It rethought the way of bandwidth sharing among applications and considers different QoS utility requirements of various applications in rate allocation. In addition, this approach assumes that the application's utility function is strictly increasing, but may not be strictly concave. As a result this approach leads to fairer rate allocation for multimedia and real-time applications.

In following subsections, we formulate rate allocation problem for SVC-encoded video streams by taking into account link capacity constraints and energy limitation of sensor nodes in the optimization problem. Firstly, we formulate the problem using NUM framework as it contains generalized cases and try to overcome its non-convexity by a transformation [18]. Then, we reformulate the problem using utility proportional fairness approach to solve the problem as it is more suitable in case of our work.

\subsection{Problem Formulation Using Network Utility Maximization}

In this subsection, we formulate resource allocation problem for SVC-encoded video streams in NUM framework. As mentioned before, the non-concavity of the utility function results in a nonconvex problem. We will address solving the optimization problem in details in subsection (4.1.A). The main goal of NUM is to achieve maximum total utility of all streams regarding to link capacity and sensor node energy constraints, so we have

$$
\underset{m_{s} \leq x \leq M_{s}}{\operatorname{maximize}} \sum_{s \in S} \tilde{U}_{s}\left(x_{s}\right)
$$

Subject to:

$$
\begin{aligned}
& \sum_{s} R_{l s} x_{s} \leq c_{l} \quad l \in L \\
& \sum_{s} E_{s} x_{s} \leq P_{s}^{\max } \quad s \in S
\end{aligned}
$$

In (6), $\tilde{U}$ is the approximated utility function which is described in subsection (2.1). To achieve more tractable mathematical model, we rewrite the optimization problem as follows [18]

$$
\underset{m_{s} \leq x \leq M_{s}}{\operatorname{maximize}} \sum_{s \in S} \log \tilde{U}_{s}\left(x_{s}\right)
$$

Subject to:

$$
\begin{aligned}
& \sum_{s} R_{l s} x_{s} \leq c_{l} \quad l \in L \\
& \sum_{s} E_{s} x_{s} \leq P_{s}^{\max } \quad s \in S
\end{aligned}
$$

In [18] was proved that equation (8) is the approximation for (6) and we can solve it instead of (6). 
International Journal of Computer Networks \& Communications (IJCNC) Vol.4, No.1, January 2012

Definition 2: A rate allocation $x=\left[x_{1}, x_{2}, \ldots, x_{S}\right]$ is feasible, if and only if $x_{s} \in\left[m_{s}, M_{s}\right]$ and total source rates at link $l$ must not exceed its capacity $\sum_{s} R_{l s} x_{s} \leq c_{l}$, also the power consumption of node $s$ should not be more than its maximum allowed power consumption $\sum_{s} E_{s} x_{s} \leq P_{s}^{\max }$.

\subsection{Problem Formulation Using Utility Proportional Fairness}

As mentioned in subsection (2.1), to deal with non-differentiable behaviour of staircase utility functions, we used the multimodal sigmoid approximation. Now, to ensure fair allocation among streams according explanation in Section 3 we use the utility-proportional optimization approach proposed by Wang et al.[8]. It is worth to mention that this approach help us to deal with the nonconcavity of the approximated utility function. By adopting utility-proportional approach, the underlying optimization problem can be formulated as

$$
\underset{m_{s} \leq x \leq M_{s}}{\operatorname{maximize}} \sum_{s=1}^{S} \int_{m_{s}}^{x_{s}} \frac{w_{s} d z}{\tilde{U}(z)}
$$

Subject to:

$$
\begin{aligned}
& \sum_{s} R_{l s} x_{s} \leq c_{l} \quad l \in L \\
& \sum_{s} E_{s} x_{s} \leq P_{s}^{\max } \quad s \in S
\end{aligned}
$$

After some elementary calculus, we have

$$
\operatorname{maximize}_{m_{s} \leq x \leq M_{s}} \sum_{s=1}^{S} \int_{m_{s}}^{x_{s}} \frac{w_{s} d z}{\tilde{U}(z)}=\sum_{s=1}^{S} \sum_{i_{s}=0}^{N-1} \int_{z \in D_{i s}} \frac{w_{s}}{F\left(z, \alpha, k_{s} i_{s}\right)+i_{s}} d z
$$

Where

$$
D_{i_{s}}=\left\{z \mid k_{s} i_{s}-\frac{k_{s}}{2} \leq z \leq \min \left(x_{s}, k_{s} i_{s}+\frac{k_{s}}{2}\right)\right\}
$$

Here $x_{s}^{*}$ denotes the optimal rate for sensor $s$ and $i_{s}^{*}$ is its associated layer.

\section{Optimal Solution}

In this section, we solve the optimization problems which were formulated in Section 3. In first subsection, we solve the non-convex primal problem (6) formulated using NUM framework and we overcome its non-convexity using a transformation. As we want to achieve a distributed solution, we address to solve the problem through its dual problem. Finally, we proposed a distributed algorithm resulted from solving dual problem. In second part, the problem formulated using UPF will solve with the same phases as the first problem.

\subsection{Network Utility Maximization Problem}

\section{A. Optimal Solution}

Solving the primal problem (8), (9) directly requires coordination among all sensor nodes to collect global information which is not feasible in real network. In order to overcome this obstacle, we utilize its dual problem. Utilizing the Lagrangian duality method, the defined 
problem can be solved separately at each sensor node and link individually using local information which leads to a distributed algorithm, eventually.

The Lagrangian dual corresponding to primal problem (8) is

$$
L(x, \lambda, \mu)=\sum_{s} \log \tilde{U}_{s}\left(x_{s}\right)-\sum_{l} \lambda_{l}\left(\sum_{s} R_{l s} x_{s}-c_{l}\right)-\sum_{s} \mu_{s}\left(\sum_{s} E_{s} x_{s}-P_{s}^{\max }\right)
$$

Where $\lambda_{l}$ is the positive Lagrangian multiplier which is interpreted as the price per unit bandwidth at link $l$; similarly, $\mu_{s}$ is the positive Lagrangian multiplier associated to energy constraint at node $s$. Therefore, there are two price vectors $\lambda \in R_{+}^{L}$ and $\mu \in R_{+}^{S}$.

As the approximated optimization problem described above is non-convex, the following transformation is used to achieve a convex one [18]

$$
\tilde{x}_{s}=e^{a x_{S}}
$$

Since the transformation is monotonic increasing, it makes sense that maximizing $L(\tilde{x}, \lambda, \mu)$ is equivalent to maximizing $L(x, \lambda, \mu)$. By applying the transformation, we have

$$
L(\tilde{x}, \lambda, \mu)=\sum_{s} \log \tilde{U}_{s}\left(\tilde{x}_{s}\right)-\sum_{l} \lambda_{l}\left(\sum_{s} \frac{R_{l s}}{\alpha} \log \tilde{x}_{s}-c_{l}\right)-\sum_{s} \mu_{s}\left(\sum_{s} \frac{E_{s}}{\alpha} \log \tilde{x}_{s}-P_{s}^{\max }\right)
$$

According to KKT theorem [20] at the optimal point $x_{s}^{*}$ the following condition should be satisfied:

$$
\begin{aligned}
& \left.\nabla_{\tilde{x}} L(\tilde{x}, \lambda, \mu)\right|_{\left(\tilde{x}^{*}, \lambda^{*}, \mu^{*}\right)}=0 \\
& \sum_{s} \frac{R_{l s}}{\alpha} \log \tilde{x}_{s}^{*} \leq c_{l}, \quad l \in L \\
& \sum_{s} \frac{E_{s}}{\alpha} \log \tilde{x}_{s}^{*} \leq P_{s}^{\max }, \quad s \in S \\
& \lambda_{l}^{*}, \mu_{s}^{*} \geq 0 \\
& \lambda_{l}^{*}\left(\sum_{s} \frac{R_{l s}}{\alpha} \log \tilde{x}_{s}^{*}-c_{l}\right)=\mathbf{0}, \mu_{s}^{*}\left(\sum_{s} \frac{E_{s}}{\alpha} \log \tilde{x}_{s}^{*}-P_{s}^{\max }\right)=\mathbf{0}
\end{aligned}
$$

In (20), 0 is a zero vector. Subsuming (16) into (17) results in

$$
\begin{aligned}
& \frac{\partial L}{\partial \tilde{x}_{s}}=\frac{d}{d \tilde{x}_{s}} \log \tilde{U}_{s}\left(\tilde{x}_{s}\right)-\frac{1}{a \tilde{x}_{s}} \sum_{l} R_{l s} \lambda_{l}-\frac{1}{a \tilde{x}_{s}} \sum_{s} E_{s} \mu_{s} \\
& =\frac{\tilde{U}_{s}^{\prime}\left(\tilde{x}_{s}\right)}{\tilde{U}_{s}\left(\tilde{x}_{s}\right)}-\frac{1}{a \tilde{x}_{s}} \sum_{l} R_{l s} \lambda_{l}-\frac{1}{a \tilde{x}_{s}} \sum_{s} E_{s} \mu_{s}= \\
& \frac{F^{\prime}\left(\tilde{x}_{s}, \alpha, k_{s i}\right)}{F\left(\tilde{x}_{s}, \alpha, k_{s i}\right)+i_{s}}-\frac{1}{a \tilde{x}_{s}} \sum_{l} R_{l s} \lambda_{l}-\frac{1}{a \tilde{x}_{s}} \sum_{s} E_{s} \mu_{s}=0
\end{aligned}
$$

We assume that the optimal point will be in $i$ th interval. Substituting $F\left(\tilde{x}_{s}, \alpha, k_{s} i_{s}\right)$ into the above equation and doing some algebraic manipulation yields 
International Journal of Computer Networks \& Communications (IJCNC) Vol.4, No.1, January 2012

$$
\frac{\frac{A_{s i}}{\left(\tilde{x}+A_{s i}\right)^{2}}}{\frac{\tilde{x}_{s}}{\tilde{x}_{s i}+A_{s i}}+i_{s}}-\frac{\lambda^{s}+\mu^{s}}{a \tilde{x}_{s}}=0
$$

Where $\lambda^{s}=\sum_{l} R_{l s} \lambda_{l}, \mu^{s}=\sum_{s} E_{s} \mu_{s}$ and $A_{s i}=e^{\alpha k_{s} i_{s}}$. Simplifying equation (22) we have

$$
\frac{A_{s i}}{\left(1+i_{s}\right)\left(\tilde{x}_{s}+B_{s i}\right)\left(\tilde{x}_{s}+A_{s i}\right)}=\frac{\lambda^{*}+\mu^{*}}{\alpha \tilde{x}_{s}}
$$

In above equation, $B_{s i}=\frac{i_{s}}{i_{s}+1} A_{s i} \cdot \tilde{x}_{s}^{*}$ can be obtained by solving following equation

$$
\tilde{x}_{s}^{2}+\left(A_{s i}+B_{s i}-\frac{\alpha A_{s i}}{\left(1+i_{s}\right)\left(\Gamma^{s}\right)}\right) \tilde{x}_{s}+A_{s i} B_{s i}=0
$$

Where $\Gamma^{s}=\lambda^{s}+\mu^{s}$.Solving the above equation will lead to obtain $\tilde{x}_{s}^{*}$ as follows

$$
\tilde{x}_{s}^{*}=A_{s i} \frac{\frac{\alpha}{\Gamma^{s}}-2 i_{s}-1+\sqrt{\left(1-\frac{\alpha}{\Gamma^{s}}\right)^{2}-\frac{4 i_{s} \alpha}{\Gamma^{s}}}}{2\left(1+i_{s}\right)}
$$

Since (25) must have a real answer, we deduce that

$$
i_{s}^{*}=\left\lfloor\frac{\Gamma^{s}}{4 \alpha}\left(1-\frac{\alpha}{\Gamma^{s}}\right)^{2}\right\rfloor
$$

Solving following equation yields optimal source rate $\tilde{x}_{s}^{*}$ as follows

$$
x_{s}^{*}=\left[\frac{1}{\alpha} \log \tilde{x}_{s}^{*}\right]_{m_{s} \leq x \leq M_{s}}
$$

Now we solve the problem through its dual. The dual problem is as follows

$$
\underset{\lambda, \mu \geq 0}{\operatorname{maximize}} D(\lambda, \mu)
$$

Where $D(\lambda, \mu)$ is dual function that is defined as follows

$$
D(\lambda, \mu)=\operatorname{minimize}_{m_{s} \leq \tilde{x} \leq M_{s}} L(\tilde{x}, \lambda, \mu)
$$

As it is obvious, problem (29) is an unconstrained optimization problem and for the dual problem we have

$$
D(\lambda, \mu)=L\left(\tilde{x}^{*}, \lambda, \mu\right)
$$

\section{B. Solving Dual Problem}

In this part we address to solve the dual problem (28) using gradient projection method [21] where link and node prices are adjusted in opposite direction to the gradients $\nabla D(\lambda)$ and $\nabla D(\mu)$ , respectively: 


$$
\lambda_{l}^{(t+1)}=\left[\lambda_{l}^{(t)}-\gamma \nabla \frac{\partial D\left(\lambda^{(t)}\right)}{\partial \lambda_{l}}\right]^{+}
$$

For the derivatives of $\nabla D(\lambda)$, we have [20]

$$
\frac{\partial D\left(\lambda^{(t)}\right)}{\partial \lambda_{l}}=c_{l}-\sum_{s} R_{l s} x_{s}^{(t)}
$$

Substituting (32) in (31), we get

$$
\lambda_{l}^{(t+1)}=\left[\lambda_{l}^{(t)}-\gamma\left(c_{l}-\sum_{s} R_{l s} x_{s}^{(t)}\right)\right]^{+}
$$

Where $\lambda^{(t)}=\left(\lambda_{l}^{(t)}, l \in L\right)$ denotes the value of $\lambda$ at $t$ th iteration.

We have the same consequenses for $\nabla D(\mu)$ as follows

$$
\mu_{s}^{(t+1)}=\left[\mu_{s}^{(t)}-\gamma \nabla \frac{\partial D\left(\mu^{(t)}\right)}{\partial \mu_{s}}\right]^{+}
$$

For the derivatives of $D(\mu)$, we have [20]

$$
\frac{\partial D\left(\mu^{(t)}\right)}{\partial \mu_{s}}=P_{s}^{\max }-\sum_{s} E_{s} x_{s}^{(t)}
$$

Substituting (35) in (34), we get

$$
\mu_{s}^{(t+1)}=\left[\mu_{s}^{(t)}-\gamma\left(P_{s}^{\max }-\sum_{s} E_{s} x_{s}^{(t)}\right)\right]^{+}
$$

Here $\gamma \succ 0$ is a small step size, $[z]^{+}=\max \{z, 0\}$ and $\mu^{(t)}=\left(\mu_{s}^{(t)}, s \in S\right)$ denote the value of $\mu$ in $t$ th iteration step. The value of $x_{s}^{(t)}$ in (33) and (36) is obtained from (27).

Using (25), (27), (33) and (36) we can achieve an iterative solution for the primal problem (6). In order to assure conversance of (33) and (36), the step size should be chosen small enough. In each iteration, dual variables $\lambda$ and $\mu$, will be updated based on their previous values and primal variable $x$. Finally, after several iterations these variables reach their optimal values. In following subsection, we propose a distributed and iterative algorithm based on obtained equations.

\section{Distributed Algorithm}

The proposed algorithm is composed of two parts:

- Link Algorithm for $t=1,2, \ldots$ :

1- Calculate the aggregate traffic which cross the link $l$ by receiving data rates of sensors that uses the link.

2- Compute a new link price using (33).

3- Communicate the new value to all sensors which use link $l$

- Node Algorithm for $t=1,2, \ldots$ : 
International Journal of Computer Networks \& Communications (IJCNC) Vol.4, No.1, January 2012

1- Calculate the aggregate energy consumption in the current time slot using equation (3).

2- Compute a new node price based on (36).

- Source algorithm

1- The aggregate link and node price are calculated.

2- Each sensor node computes its rate based on the total prices on its route using (25) and (27) equations.

These algorithms are executed repeatedly until the algorithm converges to the optimal video rates and optimal link and node prices. The distributed algorithm is represented as Algorithm 1.

\section{Algorithm 1. Rate Allocation Algorithm for SVC-Encoded Streams in WSN}

Initialization

Initialize the following items:

1. Set of sources, links, routing matrix and power matrix

2. Set $E^{s}, E^{t}$, and $E^{r}$

3. Set step size $\gamma$ and $c_{l}$ for $l \in L$ and $k_{s}$ for $s \in S$

Main Loop

Do until $\left|x_{s}^{(t+1)}-x_{s}^{(t)}\right| \prec \varepsilon$

1. For each $l \in L$, compute new link price:

$$
\lambda_{l}^{(t+1)}=\left[\lambda_{l}^{(t)}-\gamma\left(c_{l}-\sum_{s} R_{l s} x_{s}^{(t)}\right)\right]^{+}
$$

2. For each $s \in S$,compute new Power price:

$$
\mu_{s}^{(t+1)}=\left[\mu_{s}^{(t)}-\gamma\left(P_{s}^{\max }-\sum_{s} E_{s} x_{s}^{(t)}\right)\right]^{+}
$$

3. For each $s \in S$, compute new source rate:

- Calculate the total link and node price as follows:

$$
\begin{aligned}
& \lambda^{s(t)}=\sum_{l} R_{l s} \lambda_{l}^{(t)} \\
& \mu^{s(t)}=\sum_{s} E_{s} \mu_{s}^{(t)} \\
& \Gamma^{s(t)}=\lambda^{s(t)}+\mu^{s(t)}
\end{aligned}
$$

- Choose the optimal rate price as follows:

$$
\begin{gathered}
i_{s}^{(t+1)}=\left\lfloor\frac{\Gamma^{s(t)}}{4 \alpha}\left(1-\frac{\alpha}{\Gamma^{s}(t)}\right)^{2}\right\rfloor \\
\tilde{x}_{s}^{(t+1)}=A_{s i} \frac{\frac{\alpha}{\Gamma^{s(t)}}-2 i_{s}{ }^{(t+1)}-1+\sqrt{\left(1-\frac{\alpha}{\Gamma^{s(t)}}\right)^{2}-\frac{4 i_{s}{ }^{(t+1)} \alpha}{\Gamma^{s(t)}}}}{2\left(1+i_{s}{ }^{(t+1)}\right)}
\end{gathered}
$$

Where $A_{s i}=e^{\alpha k_{s} i_{s}}$

$$
x_{s}^{(t+1)}=\left[\frac{1}{\alpha} \log \tilde{x}_{s}^{(t+1)}\right]_{m . \leq x \leq M .}
$$




\subsection{Utility Proportional Fairness Solution}

\section{A. Optimal Solution}

In this subsection we address to solve the optimization problem formulated in UPF approach.

Same as the first problem, we write Lagrangian of (10) as follows

$$
L(x, \lambda, \mu)=\sum_{s} \sum_{i_{s}=0}^{N-1} \int_{z \in D_{i s}} \frac{w_{s}}{F\left(z, \alpha, k_{s} i_{s}\right)+i_{s}} d z-\sum_{l} \lambda_{l}\left(\sum_{s} R_{l s} x_{s}-c_{l}\right)-\sum_{s} \mu_{s}\left(\sum_{s} E_{s} x_{s}-P_{s}^{\max }\right) \text { (37) }
$$

As introduced before $\lambda_{l}$ and $\mu_{s}$ are positive Lagrangian multiplier related to the link capacity constraints $l$ and the energy limitation of sensor $s$.

As proved in [22], problem (10) is convex and have a unique maximizer. At optimal point,the KKT condition should be satisfied:

$$
\begin{aligned}
& \left.\nabla_{x} L(x, \lambda, \mu)\right|_{\left(x^{*}, \lambda^{*}, \mu^{*}\right)}=0 \\
& \sum_{s} R_{l s} x_{s}^{*} \leq c_{l}, \quad l \in L \\
& \sum_{s} E_{s} x_{s}^{*} \leq P_{s}^{\max }, \quad s \in S \\
& \lambda_{l}^{*}, \mu_{s}^{*} \geq 0 \\
& \lambda_{l}^{*}\left(\sum_{s} R_{l s} x_{s}^{*}-c_{l}\right)=\mathbf{0}, \mu_{s}^{*}\left(\sum_{s} E_{s} x_{s}^{*}-P_{s}^{\max }\right)=\mathbf{0}
\end{aligned}
$$

Substituting (37) in (38), we get

$$
\begin{aligned}
& \frac{\partial L}{\partial x_{s}}=\frac{w_{s}}{F\left(x_{s}, \alpha, k_{s} i_{s}\right)+i_{s}}-\sum_{l} R_{l s} \lambda_{l}-\sum_{s} E_{s} \mu_{s}= \\
& \frac{w_{s}}{\frac{1}{1+e^{-\alpha\left(x_{s}-k_{s} i_{s}\right)}}+i_{s}}-\sum_{l} R_{l s} \lambda_{l}-\sum_{s} E_{s} \mu_{s}=0
\end{aligned}
$$

After some manipulations, we can obtain the optimal rate from following equation

$$
x_{s}^{*}=\left[k_{s} i_{s}^{*}-\frac{1}{\alpha} \log \left(\frac{\Gamma^{s}}{w_{s}-\Gamma^{S} i_{s}^{*}}-1\right)\right]_{m_{s}}^{M_{s}}
$$

Where $\Gamma^{s}$ defined in subsection (4.1.A). As the argument of the logarithm should be positive, $i_{s}^{*}$ should satisfy $\frac{\Gamma^{s}}{w_{s}-\Gamma^{s} i_{s}^{*}} \succ 1$ which lead to

$$
i_{s}^{*}=\left\lceil\frac{w_{s}-\Gamma^{s}}{\Gamma^{s}}\right\rceil
$$

To achieve a distributed approach, the dual problem should be solved. For the dual problem, we have 
International Journal of Computer Networks \& Communications (IJCNC) Vol.4, No.1, January 2012

$$
\underset{\lambda, \mu \geq 0}{\operatorname{minimum}} D(\lambda, \mu)
$$

$D(\lambda, \mu)$ is the dual function which is defined as following

$$
D(\lambda, \mu)=\operatorname{maximum}_{m_{s} \leq x \leq M_{s}} L(x, \lambda, \mu)
$$

As the unconstrained problem (46) is already solved by $x^{*}$, so we get

$$
D(\lambda, \mu)=L\left(x^{*}, \lambda, \mu\right)
$$

Where the optimal rate $x^{*}$ is obtained by (43).

\section{B. Solving Dual Problem}

In this part, we address solving the dual problem (45). In this case, the strict convexity of the primal problem (10) insures the strong duality. So, we can conclude that we obtain optimal solution for the primal problem by solving dual problem [20].

Similar to subsecion (4.1.A), we solve the dual problem (45) using gradient pojection approach [21]. As a result, we get the same iterative equations as (33) and (36) respectively for dual variables $\lambda$ and $\mu$ which help us to calculate their value at $t$ th iteration step. We should noice that in these equation, $x_{s}^{(t)}$ is given by (43).

In following subsection, we propose a distributed algorithm based on the iterative equations (33) and (36) to calculate new link and node prices at each iteration step, respectively. Then using new rate is calculated based on the total prices $\Gamma^{s}$ according to (43) and (44).

\section{Distributed Algorithm}

Same as Algorithm 1, second algorithm is also consisted of three parts thus we refuse to explain them again. But we should notice that the main difference of these algorithms is the source algorithm. In fact, Algorithm 2 allocates rates to video streams according their QoS requirements. 


\section{Algorithm 2. Rate Allocation Algorithm for Scalable Multimedia in WSNs Using UPF}

Initialization

Initialize the following items:

1. Set sources, links, routing matrix and power matrix

2. Set $E^{s}, E^{t}$, and $E^{r}$

3. Set step size $\gamma$ and $c_{l}$ for $l \in L$ and $w_{s}$ for $s \in S$

Main Loop

Do until $\left|x_{s}^{(t+1)}-x_{s}^{(t)}\right| \prec \varepsilon$

1. For each $l \in L$, compute new link price according to

$$
\lambda_{l}^{(t+1)}=\left[\lambda_{l}^{(t)}-\gamma\left(c_{l}-\sum_{s} R_{l s} x_{s}^{(t)}\right)\right]^{+}
$$

2. For each $s \in S$, compute new Power price as follows

$$
\mu_{s}^{(t+1)}=\left[\mu_{s}^{(t)}-\gamma\left(P_{s}^{\max }-\sum_{s} E_{s} x_{s}{ }^{(t)}\right)\right]^{+}
$$

3. For each $s \in S$, compute new source rate:

- Calculate the total link and node price as follows:

$$
\begin{aligned}
& \lambda^{s(t)}=\sum_{l} R_{l s} \lambda_{l}^{(t)} \\
& \mu^{s(t)}=\sum_{s} E_{s} \mu_{s}^{(t)} \\
& \Gamma^{s(t)}=\lambda^{s(t)}+\mu^{s(t)}
\end{aligned}
$$

- $\quad$ calculate the optimal rate price as follows

$$
\begin{gathered}
i_{s}^{(t+1)}=\left\lceil\frac{w_{s}-\Gamma^{s(t)}}{\Gamma^{s(t)}}\right\rceil \\
x_{s}^{(t+1)}=\left[k_{s} i_{s}^{(t+1)}-\frac{1}{\alpha} \log \left(\frac{\Gamma^{s(t)}}{w_{s}-\Gamma^{(t)} i_{s}^{(t+1)}}-1\right)\right]_{m_{s}}^{M_{s}}
\end{gathered}
$$

\section{Simulation Results}

In this section, we present simulation results using MATLAB to illustrate the merits of the proposed algorithms. The topology of the wireless sensor network considered in this paper is demonstated in Figure 2 where eight sensor nodes and a sink are deployed. There are eight links which their capacities are set as $\left(c_{1}, \ldots, c_{8}\right)=(18,18,15,10,12,10,10,10)$ Mbps. Each sensor node transmits its sensed data through a unique route; routes are as follows $L_{1}=\left\{l_{1}\right\}, L_{2}=\left\{l_{2}\right\}$, $L_{3}=\left\{l_{3}, l_{1}\right\}, L_{4}=\left\{l_{4}, l_{2}\right\}, L_{5}=\left\{l_{5}, l_{2}\right\}, L_{6}=\left\{l_{6}, l_{3}, l_{1}\right\}, L_{7}=\left\{l_{7}, l_{3}, l_{1}\right\}, L_{8}=\left\{l_{8}, l_{5}, l_{2}\right\}$. Since close nodes to sink such as $s_{1}$ and $s_{2}$ relatively pass heavier traffic than other nodes, we allocate more resource to them. The parameters of the power dissipation model $E^{s}, E^{t}$ and $E^{r}$ are set as 100 $\mathrm{nj} / \mathrm{bit}, 150 \mathrm{nj} / \mathrm{bit}$ and $158 \mathrm{nj} / \mathrm{bit}$ based on the popular CC2420 [23] RF transceiver power dissipation measurements, respectively. Maximum node power consumpion is se to $5 \mathrm{~mW}$. 
Sensor nodes according to their QoS requirements choose different values of $k_{s}$ which define as follow: $\left(k_{1}, \ldots, k_{8}\right)=(1.2,1.3,1,1,1.1,8,8,7, .7)$.In addition, the stepsize is set to be $\gamma=0.007$ and the parameter $\alpha$ for all sensor nodes is chosen to be 8 .

In Algorithm 2, the weight factor is not equal for all sensors and are set to be $\left(w_{1}, \ldots, w_{8}\right)=(1.4,1.2, .75,1, .75, .5, .5, .75)$. Other parameters are identical in both algorithms.

In the simulation study, we investigate how fast the algorithms converge to the suboptimal values. So, the evolution of source rates for Algrithm 1 is depiced in Figure 3. Also, the evolution of link prices are depicted in Figure 4 for first algorithm. As it is obvious, both figures converge to the steady state in less than 20 iterations which show the effictiveness of our algorithms. As the dual node prices converge to zero, its corresponding figure is not depicted here due to space limitation.

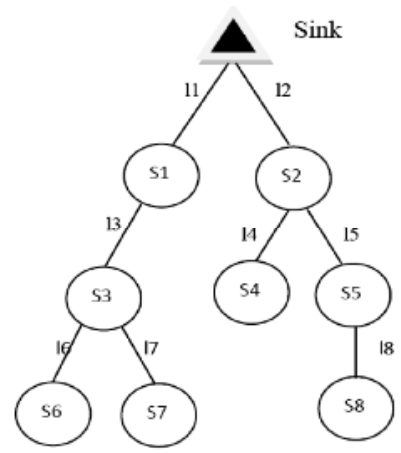

Figure 2: Topology of WSN

As illustrated in Figure 3, $s_{1}$ and $s_{2}$ recieve more bandwith, it makes sense, since they act as relay nodes for passing the traffic of other nodes. Also, they can transmit their data more efficiently than other nodes as they have direct route to the sink. Figure 4 shows the dual link price status, two links $l_{1}$ and $l_{2}$ have saturated while other links still have capacity to pass traffics. 


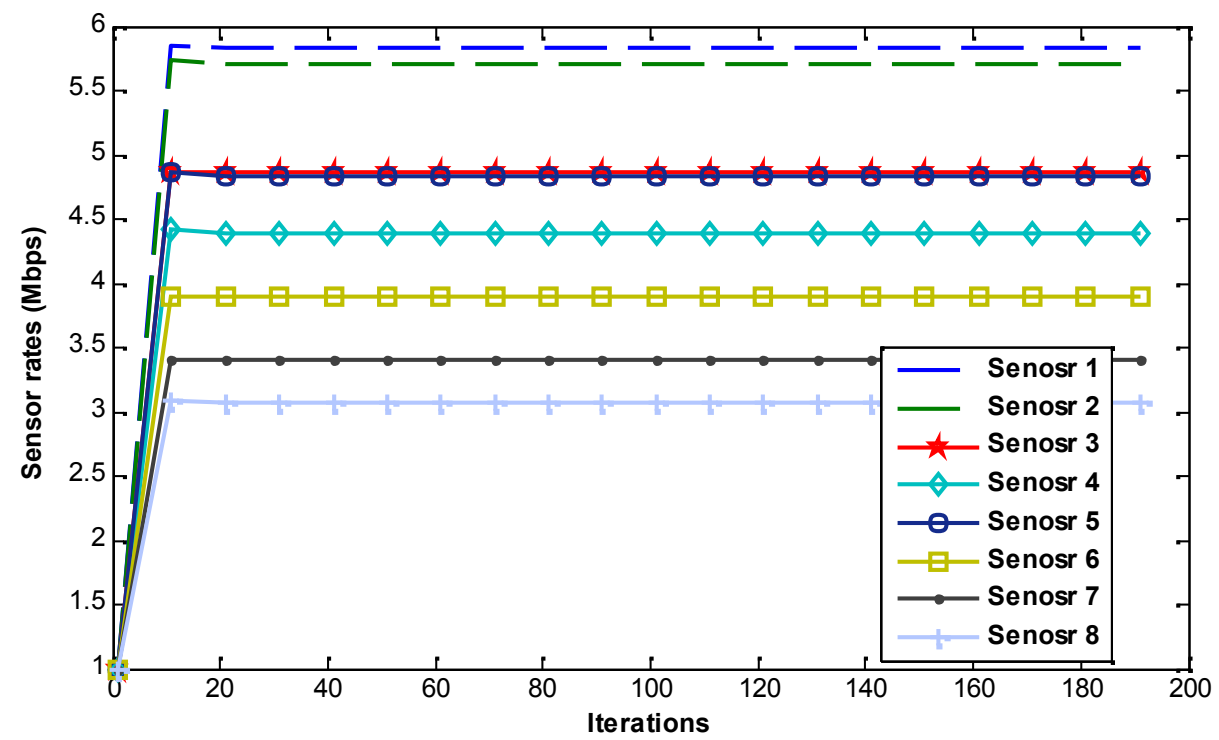

Figure 3: Evaluation of Source rates in NUM Approach

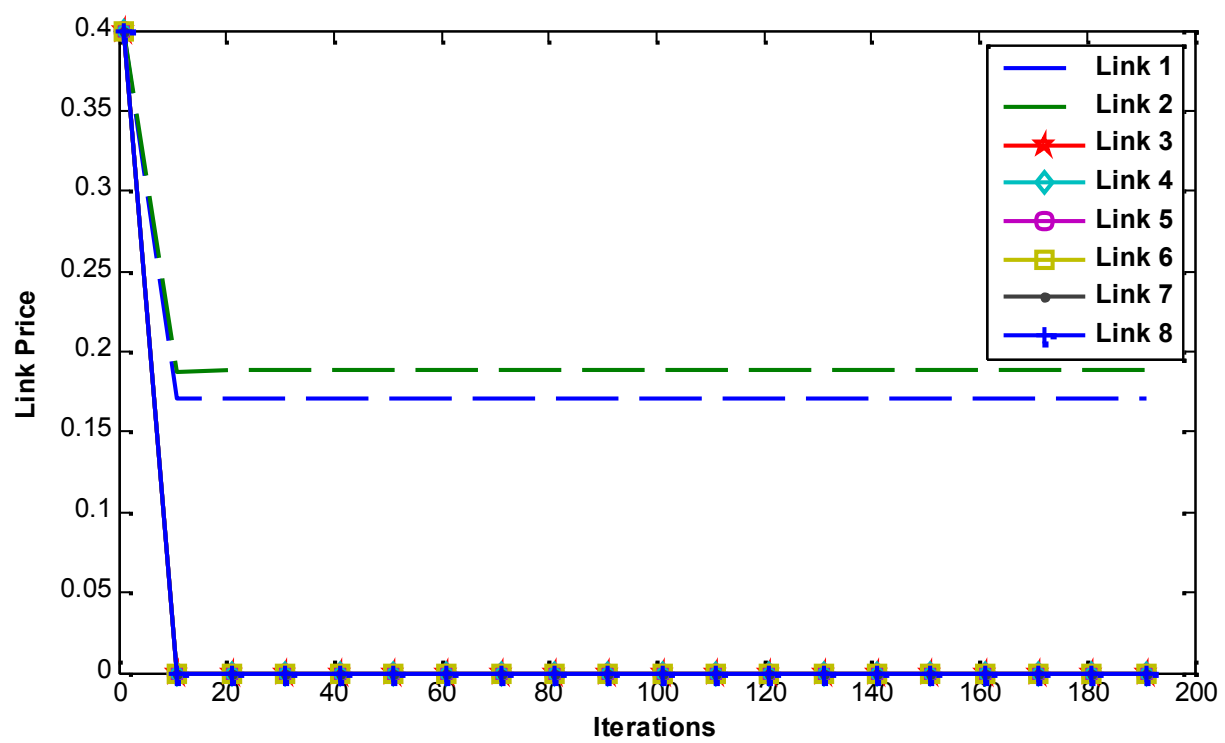

Figure 4: Evaluation of Link Prices in NUM Approach

The evolution of source rates and link price $\left(\lambda_{l}\right)$ for Algrithm 2 are depiced in Figure 5 and 6 , respectively. In this case, after about 10 iterations, the algorithm passes transient state and converges to the steady state. As mentioned before, $k_{s}$ denotes the rate and qualiy requirment for each video stream, i.e. the higher value is more demanded. It is noticeble that UPF allocate greater rates to sensors that have a larger $k_{s}$ and this is in accordance by its theory which allocate rate based on QoS requiments of streams. 


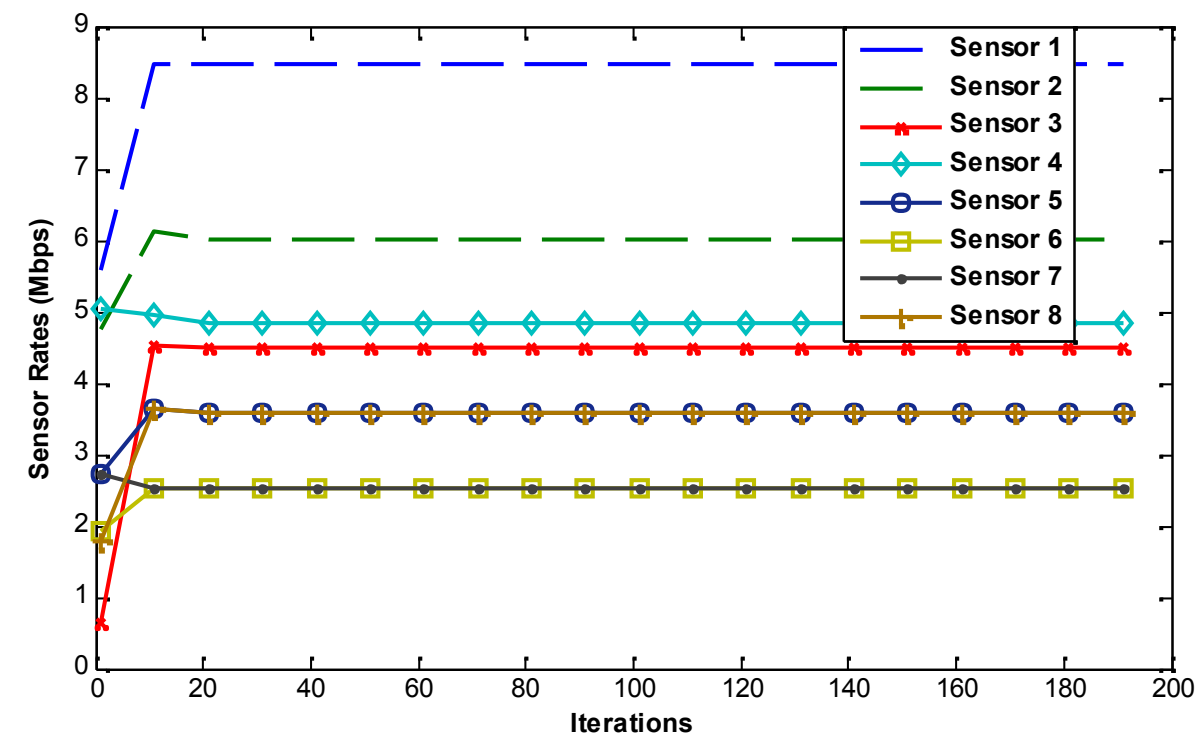

Figure 5: Evaluation of Source Rates in UPF Approach

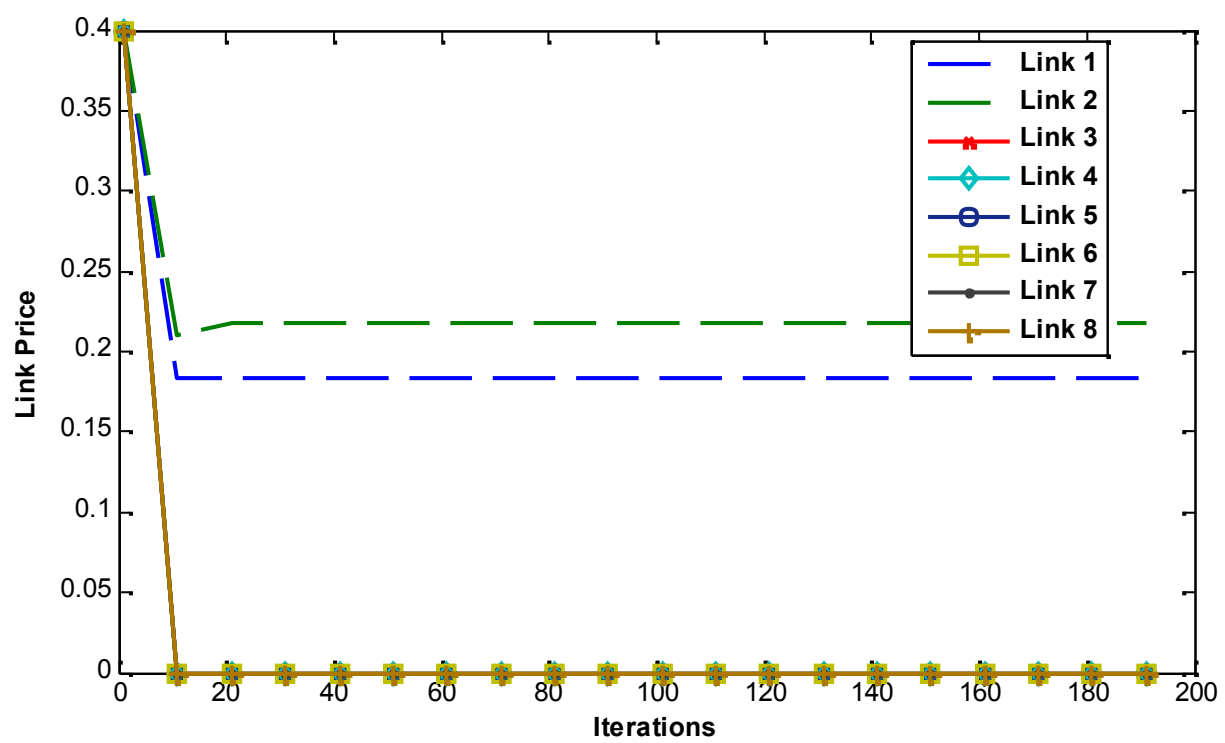

Figure 6: Evaluation of Link Prices in UPF Approach

Here, the values of $w_{s}$ determine the priority of rate allocation which makes the results of allocated bandwith totally different. The traffic of other nodes is transmitted through $l_{1}$ and $l_{2}$, so it is resonable that they have non-zero values and other links converge to zero (Figure 6).

It is worth to mention that the various values in $w_{s}, k_{s}, c_{l}$ and also power dissipation result in different rate allocation, but in all of the cases these two algorithms will converge to the steady state repidly. 


\section{Conclusions}

In this paper, a resource allocation problem for SVC-encoded streams in WSNs is addressed. This problem is formulated by applying two different fairness approach including Network Utility Maximization and Utility Proportional Fairness which result in two various optimization problems. Our optimization problem has two constraints: link capacity and energy limitation of sensor node. The latter one is added to ensure defined lifetime for WSN. Each optimization problem is solved through dual problem using gradient projection method. We achieved two distributed algorithm for resource allocation by solving these optimization problems. Finally, the convergence and efficiency of algorithms are verified by simulation results.

In this paper, we assume the capacity of wireless links are fix and also we suppose the identical energy dissipation model for sensor nodes. As a future works, we can consider different link capacity and distinct energy model. In addition, the mathematical optimization model can be easily extended to address heterogeneous sensor networks where there exist different traffics besides video streams. Here, we assume a single route between each sensor node and sink instead the resource allocation problems can be evaluated in a multipath network as an extension to this work.

\section{REFERENCES}

[1] H. Schwarz, D. Marpe, and T. Wiegand, "Overview of the scalable video coding extension of H.264/AVC," IEEE Trans. Circuits and Systems for Video Tech., vol. 17, no. 9, pp. 1103-1120, Sep. 2007.

[2] Advanced Video Coding for Generic Audiovisual Services, ITU-T Rec. H.264 and ISO/IEC 1449610 AVC, Amendment 3: Scalable Video Coding, v3: 2005.

[3] Advanced Video Coding for Generic Audiovisual Services, ITU-T Recommendation H.264 and ISO/IEC 14496-10 AVC, v3: 2005.

[4] The ITU-T H.264 Standard and the ISO/IEC MPEG-4 Part 10 Standard (ISO/IEC 14496-10), May 2003.

[5] Yick J., Mukherjee B., and Ghosal D, "Wireless sensor network survey", Computer Networks Elsevier, pp.2292-2330, 2008.

[6] F. P. Kelly, A. Maulloo, and D. Tan, "Rate control for communication networks: shadow prices, proportional fairness and stability," J. Op. Res. Soc., vol.49, pp. 237-252, Mar. 1998.

[7] S. H. Low, and D. E. Lapsley, "Optimal flow control, I: basic algorithm and convergence," IEEE/ACM Trans. Netw., vol. 7, no. 6, pp. 861-874, Dec. 1999.

[8] W. H. Wang, M. Palaniswami, and S. H. Low, "Application oriented flow control: fundamentals, algorithms and fairness," IEEE/ACM Trans.Netw., vol.14, no.6, pp.1282-1291, Dec. 2006.

[9] M. Chiang, S. H. Low, A. R. Calderbank, and J. C. Doyle, "Layering as optimization decomposition: A mathematical theory of network architectures," In Proc. of the IEEE, vol. 95, no. 1, pp. 255-312, 2007.

[10] S. Li, W. Sun, Y. Zhang and H. Zhang, "An Optimal Rate and Routing Scheme for Multipath Networks," International Journal of Computers Communications \& Control, vol. 6, no. 4, pp. 657668, 2011.

[11] J. J. Jaramillo, R. Srikant, and L. Ying, "Scheduling for optimal rate allocation in ad hoc networks with heterogeneous delay constraints," IEEE J. Sel. Areas Comm., vol. 29, no. 5, pp. 979-987, May 2011.

[12] M. Zheng, H. Yu, J. Zheng, et al. "Tradeoff between utility and lifetime in energy-constrained wireless sensor networks," Journal of Control Theory and Application, pp. 75 - 80, 2010. 
International Journal of Computer Networks \& Communications (IJCNC) Vol.4, No.1, January 2012

[13] J. Chen, W. Xu, S. He, Y. Sun, P. Thulasiramanz, and X. Shen. "Utility-Based Asynchronous Flow Control Algorithm for Wireless Sensor Networks". IEEE J. Sel. Areas Comm., vol. 28, no. 7, pp. 1116-1126, 2010.

[14] J. Jin, W. H. Wang, and M. Palaniswami, "Application-oriented flow control for wireless sensor networks," 3rd International Conference on Networking and Services, Athens, Greece, pp. 423-429, 2007.

[15] L. Su, Y. Gao, Y. Yang, and G. Cao. "Towards optimal rate allocation for data aggregation in wireless sensor networks," In the 12th ACM International Symposium on Mobile AdHoc Networking and Computing, (Mobihoc'11), Paris, France, 2011.

[16] S. Shenker, "Fundamental design issues for the future Internet," IEEE J. Sel. Areas Comm., vol.13, no. 7, pp. 1176-1188, Sep. 1995.

[17] P. Hande, Sh. Zhang, M. Chiang, "Distributed Rate Allocation for Inelastic Flows", IEEE/ACM Trans.Netw., Feb. 2008.

[18] M.S. Talebi, A. Khonsari, M.H. Hajiesmaili and S. Jafarpour, "A Suboptimal network utility maximization approach for scalable multimedia applications," IEEE GLOBEOCOM, Honolulu, HI, 2009.

[19] W. Heinzelman, A. Chandrakasan, and H.Balakrishnan, "An application-specific protocol architecture for wireless micro sensor networks, "IEEE Trans. Wireless Comm., vol. 1, no. 4, pp. 660-670, 2002.

[20] S. Boyd, and L.Vandenberghe, “Convex Optimization”, Cambridge University Press, 2003.

[21] D. Bertsekas, "Nonlinear Programming”, Athena Scientific, 1999.

[22] M.S. Talebi, A. Khonsari, and M.H. Hajiesmaili, "Optimization bandwidth sharing for multimedia transmission supporting scalable video coding," In Local Computer Networks, pp. 185 -192, Oct. 2009.

[23] Chipcon Inc. CC2420, True single-chip 2.4 GHz IEEE 802.15.4/ZigBee RF transceiver with MAC support. http:// www.chipcon.com

\section{Authors}

Seyedeh Zahra Sdari Tabaee Zavareh received the B.S. degree in Hardware Engineering from Azad University of South Branch, Tehran, Iran, in 2004, the M.S. degree in computer architecture from Azad University Science and Research Branch, Tehran, Iran, in 2011. Her research interests include wireless communication and networks, with a focus on optimization and cross layer for wireless sensor networks, distributed resource allocation and multimedia transmission over wireless networks.

Mahmood Fathy received the B.S. degree in Electronics from Iran University of Science and Technology, Tehran, Iran, in 1985, the M.S. degree in computer architecture from Bradford University, West Yorkshire, U.K., in 1987, and the Ph.D. degree in image processing computer architecture from the university of Manchester Institute of Science and Technology, Manchester, U.K., in 1991. Since 1991, he has been an Associate Professor with the Department of Computer Engineering, Iran University of Science and Technology. His research interests include the quality of service in computer networks, including video and image transmission over Internet, the applications of vehicular ad hoc networks in intelligent transportation systems, and real-time image processing, with particular interest in traffic engineering. 